\title{
DISSECTION OF THE HYPERCUBE INTO SIMPLEXES
}

\author{
D. G. MEAD
}

\begin{abstract}
A generalization of Sperner's Lemma is proved and, using extensions of $p$-adic valuations to the real numbers, it is shown that the unit hypercube in $n$ dimensions can be divided into $m$ simplexes all of equal hypervolume if and only if $m$ is a multiple of $n !$. This extends the corresponding result for $n=2$ of Paul Monsky.
\end{abstract}

The question as to whether a square can be divided into an odd number of (nonoverlapping) triangles all of the same area was answered in the negative by Thomas [3] (if all the vertices of the triangles are rational numbers), and in general by Monsky [2].

In this note we generalize Monsky's result to $n$ dimensions and prove the following:

Theorem. Let $C$ be the unit hypercube in $n$ dimensions. Then $C$ can be divided into $m$ simplexes all of equal hypervolume if and only if $m$ is a multiple of $n !$.

The proof is divided into two parts. In the first we obtain a slight generalization of Sperner's Lemma while the second employs extensions of $p$-adic valuations to the real numbers.

Let $R$ be an $n$-polytope in $n$-space. A simplicial decomposition of $R$ is a division of $R$ into simplexes such that if $v$ is a vertex of some simplex on the boundary of the simplex $S$, then $v$ is a vertex of $S$. We consider a simplicial decomposition of $R$ in which each vertex of a simplex is labeled $p_{i}$ for some $i$, $0<i<n$, and we call the $k$-simplex $S$ a complete $k$-simplex if the vertices of $S$ are labeled $p_{0}, p_{1}, \ldots, p_{k}$.

LEMMA 1 (SPERNER's LemMa). Consider a simplicial decomposition of an $n$-polytope $R$ in which each vertex is labeled $p_{i}, 0<i<n$. Then the number of complete $n$-simplexes is odd if and only if the number of complete $(n-1)-$ simplexes on the boundary of $R$ is odd.

Proof. Note that every complete $(n-1)$-simplex on the boundary of $R$ occurs in one $n$-simplex while all other complete $(n-1)$-simplexes occur in two $n$-simplexes. Also, a complete $n$-simplex has precisely one complete $n-1$ dimensional face, while an "incomplete" $n$-simplex has 0 or 2 such faces.

Received by the editors September 25, 1978.

AMS (MOS) subject classifications (1970). Primary 50B30; Secondary 12B99.

Key words and phrases. Sperner's Lemma, p-adic valuation, dissection of a unit hypercube into simplexes. 
From this, the conclusion of Sperner's Lemma follows easily.

We now consider decomposition of an $n$-polytope into simplexes, which may not be simplicial (i.e. a vertex of one simplex may be on a face, but not a vertex, of an adjacent simplex).

Lemma 2. Consider a decomposition of an n-polytope $R$ into simplexes in which each vertex is labeled $p_{i}, 0<i<n$, and such that any $k$-dimensional affine subspace which contains vertices labeled $p_{i}$, for all $0<i<k$ contains no vertex labeled $p_{i}$ with $i>k$. Then the number of complete n-simplexes is odd if and only if the number of complete $(n-1)$-simplexes on the boundary of $R$ is odd.

The proof is by induction on $n$. Since for $n=0,1$, any decomposition into simplexes is a simplicial decomposition, the result follows from Sperner's Lemma.

Assume the result for $k$-polytopes with $k<n$. We first show that the number of simplexes in the decomposition of $R$ which have a complete $(n-1)$-simplex as a face internal to $R$ is even. Let $T$ be an $(n-1)$-polytope interior to $R$ such that all the vertices of $T$ are vertices of simplexes on both sides of the hyperplane of $T$. Then $T$ can be considered as the union of two sets of $(n-1)$-simplexes from the decomposition of $R$ on the two sides of $T$. By induction, the number of complete $(n-1)$-simplexes in these two decompositions have the same parity. This shows that the number of simplexes which have a complete $(n-1)$-simplex as a face internal to $R$ is even and the proof of the lemma is completed as was the proof of Sperner's Lemma.

Turning now to the $p$-adic valuations we use $|x|_{p}$ to represent the valuations at $x$ and recall that if $x$ is rational, $x=p^{t}(a / b)$ where $(a, p)=(b, p)=$ 1 then $|x|_{p}=p^{-t}$, while $|0|_{p}=0$. It is easy to show that if $x$ and $y$ are rational $|x y|_{p}=|x|_{p}|y|_{p}$ and $|x+y|_{p}<\max \left(|x|_{p},|y|_{p}\right)$ with equality if $|x|_{p} \neq|y|_{p}$. It is known that this $p$-adic valuation can be extended to the reals (Theorem $1.2,[1$, p. 121]) and we use the same notation for the extension.

Fix the prime $p$, let $\|x\|=|x|_{p}$, and separate the points $\left(x_{1}, \ldots, x_{n}\right)$ in space into $n+1$ sets $P_{0}, P_{1}, \ldots, P_{n}$ as follows:

$$
\begin{gathered}
\left(x_{1}, \ldots, x_{n}\right) \in P_{0} \text { if }\left\|x_{i}\right\|<1 \text { for all } i, \\
\left(x_{1}, \ldots, x_{n}\right) \in P_{k} \quad \text { if }\left\|x_{k}\right\| \geqslant 1,\left\|x_{k}\right\|>\left\|x_{i}\right\| \text { for } i<k \\
\text { and }\left\|x_{k}\right\| \geqslant\left\|x_{i}\right\| \text { for } k<i .
\end{gathered}
$$

Suppose $\left(x_{1}, \ldots, x_{n}\right) \notin P_{0}$ and $k \geqslant 1$. Then $\left(x_{1}, \ldots, x_{n}\right) \in P_{k}$ if $\left\|x_{k}\right\|=$ $\max \left\|x_{j}\right\|$, and $k$ is the smallest index for which this equality holds. Note that if $\left(x_{1}, \ldots, x_{n}\right) \in P_{k}$ with $k \neq 0$ then $\left\|x_{k}\right\| \geqslant 1$. It follows easily that each $P_{k}$ is stable under translation by elements of $P_{0}$.

We next show that a $k$-dimensional affine subspace cannot contain points from each $P_{i}, 0 \leqslant i \leqslant k$, and from some additional $P_{l}$. Suppose the contrary, and let the point from $P_{j}$ have coordinates $\left(x_{1 j}, \ldots, x_{n j}\right)$. (By the above we may, and do, assume the origin is the point in $P_{0}$.) However the $p$-adic 
valuation of the determinant

$$
\left[\begin{array}{cccc}
x_{11} & \cdots & x_{k 1} & x_{l 1} \\
x_{12} & \cdots & x_{k 2} & x_{l 2} \\
\vdots & \vdots & \vdots & \\
x_{1 k} & \cdots & x_{k k} & x_{l k} \\
x_{1 l} & \cdots & x_{k l} & x_{l l}
\end{array}\right]
$$

is the same as $\left\|x_{11} x_{22} \ldots x_{k k} x_{l l}\right\|$ because it has the largest value of all terms in the expansion. Thus, the determinant is not zero and this contradicts our assumption and shows that the $\boldsymbol{P}_{\boldsymbol{i}}$ define a labelling satisfying the hypothesis of Lemma 2. Using the same idea again, we see that if $\alpha$ is the highest power of the prime $p$ which divides $n$ !, then the $p$-adic valuation of the hypervolume of a complete $n$-simplex is greater than or equal to $\alpha$ since $\left|x_{11} \ldots x_{n n}\right|_{p}>1$ and the hypervolume of the simplex is $(1 / n !) \operatorname{det}\left(x_{i j}\right)$ where $x_{i j}$ is the $i$ th coordinate of the point in $\boldsymbol{P}_{\boldsymbol{j}}$. (We assume that the origin is the point in $\boldsymbol{P}_{\mathbf{0}}$.)

Finally, since for each $k$ there is only one $k$-dimensional face of the unit hypercube $C$ which contains points from $P_{0}, P_{1}, \ldots, P_{k}$, it follows easily from Lemma 2 that if the unit hypercube $C$ is divided into $m$ simplexes, there must be an odd number of complete $n$-simplexes. Let $S$ be a complete $n$-simplex. Then the hypervolume of $S$ is $1 / m$ and from the above $|S|_{p}=|1 / m|_{p}>\alpha$ where $\alpha$ is the highest power of $p$ dividing $n !$. That is, $m=p^{\alpha} \cdot t$ for some integer $t$. Since this is true for all primes $p, m$ must be a multiple of $n !$. It is clear that one can realize any multiple of $n$ !: divide $C$ into $n$ ! simplexes of the same volume, and take the decomposition obtained by dividing a one-dimensional face of each simplex into $l$ equal parts, which yields $l \cdot n !$ simplexes of equal volume. This completes the proof of the theorem.

\section{REFERENCES}

1. George Bachman, Introduction to p-adic numbers and valuation theory, Academic Press, New York, 1964.

2. Paul Monsky, On dividing a square into triangles, Amer. Math. Monthly 77 (1970), 161-164.

3. John Thomas, A dissection problem, Math. Mag. 41 (1968), 187-190.

Department of Mathematics, University of California, Davis, Calufornia 95616 